\title{
NOUVELLE FILAIRE PARASITE DES ARTÈRES DE L'HELODERMA SUSPECTUM COPE : MACDONALDIUS ANDERSONI N. SP. (NEMATODES, ONCHOCERCIDAE)
}

\author{
Par Alain G. CHABAUD et Werner FRANK
}

Nous avons décrit très récemment (1961), chez différents Pythons vivant en captivité au Jardin Zoologique de Stuttgart, une nouvelle espèce de Filaire, Macdonaldius oschei, qui vit dans les artères et forme des pelotons qui peuvent obturer celles-ci.

Une curieuse coïncidence fait qu'une seconde espèce, particulièrement proche de la précédente, et ayant la même localisation intraartérielle, vient d'être découverte chez un autre Reptile du même Pare Zoologique. L'hôte est un spécimen d'Heloderma suspectum (distribution : Sud-Ouest des Etats-Unis et Nord-Ouest du Mexique).

Pendant les derniers jours avant la mort, il a manifesté une apathie complète. La tête était penchée obliquement par une contracture permanente. L'examen du sang a montré de très nombreuses microfilaires. L'animal est mort le 2 janvier 1961 et l'autopsie a montré la présence de Filaires adultes dans l'aorte descendante (au niveau des reins) et dans certaines grosses artères abdominales (iliaque ou rénale ?), qui paraissaient obturées. C'est le matériel ainsi obtenu que nous nous proposons de décrire :

\section{Description}

Hôte : Heloderma suspectum Cope 1869, mort en captivité au Jardin Zoologique de Stuttgart.

Localisation DES adultes: Grosses artères abdominales.

MAtÉRIEL: Un mâle holotype, une femelle allotype, deux femelles éclatées et trois gouttes épaisses de sang contenant des microfilaires.

Corps blanc, cylindrique, assez trapu pour une Filaire, recouvert d'une cuticule à stries transversales très fines et rapprochées $(1,5 \mu)$. Il n'y a pas de véritables ailes latérales, mais la cuticule est un peu 

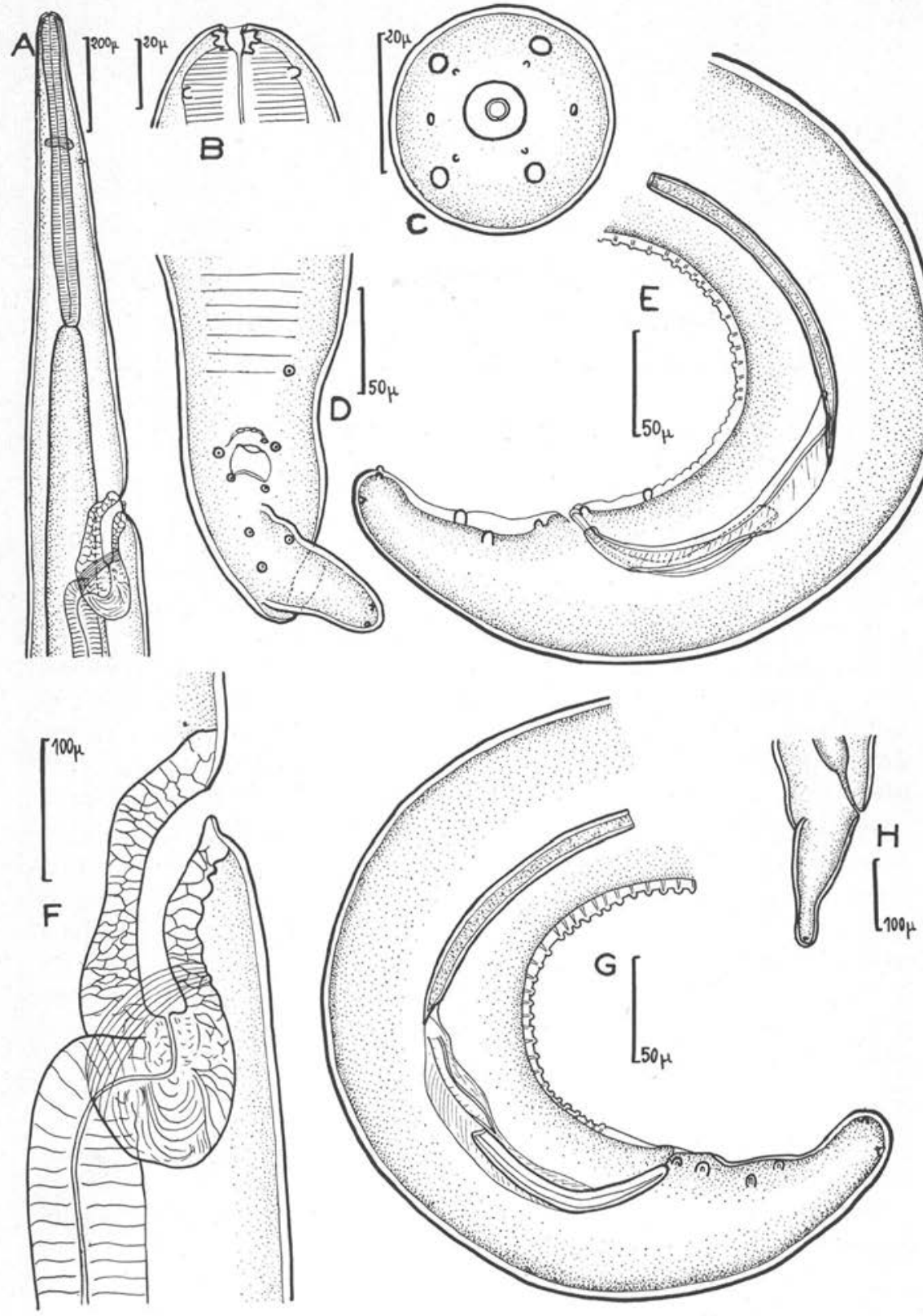

Fig. 1. - Macdonaldius andersoni

a) Extrémité antérieure, femelle, vue latérale

b) Tête, femelle, vue médiane.

c) Tête, femelle, vue apicale

d) Queue, mâle, vue ventrale

e) Extrémité postérieure, mâle, vue latérale gauche

$f$ ) Vulve et sphincter, vue latérale

g) Extrémité postérieure, mâle, vue latérale droite

h) Queue femelle, vue latérale 
dilatée sur les lignes latérales dans la portion antérieure du corps (jusqu'au niveau de la vulve chez la femelle). Tête arrondie, dépourvue de plaque ou de bouclier chitinoïde. Boưche petite, circulaire ; capsule buccale relativement grande (diamètre externe maximum : $14 \mu$, hauteur : $7 \mu$ ), avec paroi externe irrégulière donnant à la capsule un aspect étoilé (fig. $1 \mathrm{~B}$ ). Huit papilles et deux amphides. Les quatre papilles latéro-médianes plus grandes, plus saillantes et plus postérieures que les médio-médianes correspondantes. Amphides au même niveau que les papilles latéro-médianes. CEsophage cylindrique, grêle et court, non divisé. Intestin plus large que l'œsophage. Anneau nerveux situé à peu près au niveau du tiers antérieur de l'œsophage. Pore excréteur un peu en arrière de l'anneau nerveux. Diérides non perceptibles.

Mâle : Corps long de $22 \mathrm{~mm}$., large au maximum de $210 \mu$. OEsophage long de $635 \mu$, anneau nerveux et pore excréteur respectivement à 240 et $300 \mu$ de l'apex. Le tiers postérieur du corps s'atténue régulièrement jusqu'à l'extrémité postérieure qui forme deux tours de spire. La cuticule antérieure au cloaque, sur une hauteur de $2,9 \mathrm{~mm}$., qui correspond à peu près à la zone enroulée en spirale, porte des séries de crêtes transversales surajoutées à la fine striation normale. Ces crêtes, qui n'intéressent que la face ventrale, sont bien saillantes et sont espacées d'environ $7 \mu$. La queue, longue de $200 \mu$, est conique, arrondie. Elle est dépourvue d'ailes et porte sur notre spécimen huit papilles asymétriques assez grosses, plus un groupe terminal comprenant une paire de phasmides latérales et une paire de petites papilles subventrales. Au niveau du cloaque, se trouvent la première papille droite et la deuxième papille gauche ; en arrière du cloaque, la deuxième papille droite et la troisième gauche ; en avant du cloaque, une bosselure arrondie porte peut-être deux ou trois petites papilles supplémentaires. La première papille gauche est antérieure, au niveau de la première crête cuticulaire transversale ; les autres papilles $\left(3^{\circ}\right.$ et $4^{\circ}$ droites et $4^{\circ}$ gauche) sont à la partie moyenne de la queue. Gubernaculum absent. Spicules inégaux et dissemblables. Le droit, long de $110 \mu$, est concave et n'est pas nettement divisé en manche et lame. Le gauche, long de $310 \mu$, comprend un manche de $150 \mu$ et une lame formée d'un axe chitinoïde et d'ailes largement développées.

Femelle: Corps long de $50 \mathrm{~mm}$., large au maximum de $350 \mu$. Esophage long de $640 \mu$. Anneau nerveux et pore excréteur, respectivement à 280 et $320 \mu$ de l'apex. La vulve s'ouvre à $1.030 \mu$ de l'apex, au fond d'une dépression cuticulaire. Il existe un gros sphincter pyriforme, long de $300 \mu$, puis un ovéjecteur impair très long

Ann. de Parasitologie, T. XXXVI, $\mathrm{N}^{\circ} 1-2 .-1961$. 
$(3,5 \mathrm{~mm}$.$) , qui descend vers l'arrière en se dilatant progressivement,$ puis se divise en deux utérus pleins de microfilaires. Les utérus descendent parallèlemient jusqu'à l'extrémité postérieure, où ils s'abouchent aux oviductes. Les oviductes et les ovaires sont enroulés dans la portion toute postérieure du corps. La queue est assez courte $(185 \mu)$ et conique, avec une pointe fine et arrondie, qui porte deux phasmides subterminales.

Microfilaires: Des gouttes épaisses prélevées quelques jours avant la mort de l'animal ont été colorées au Giemsa, avec séchage et fixation, selon la technique indiquée par Golvan (1957), Les dimensions moyennes mesurées sur 10 spécimens sont :

Longueur de corps (sans la gaine) : $218 \mu$ (maximum : 230, minimum : 205).

Largeur du corps (sans la gaine) : 4,5 $\mu$.

Espace (céphalique) : $11 \mu$.

Anneau nerveux à $62 \mu$ de l'extrémité antérieure.

Pore excréteur à $86 \mu$ de l'extrémité antérieure.

Cellule excrétrice à $105 \mu$ de l'extrémité antérieure.

Corps interne long d'environ $16 \mu$ avec partie centrale à $150 \mu$ de l'extrémité antérieure.

Pore anal à $28 \mu$ de l'extrémité postérieure.

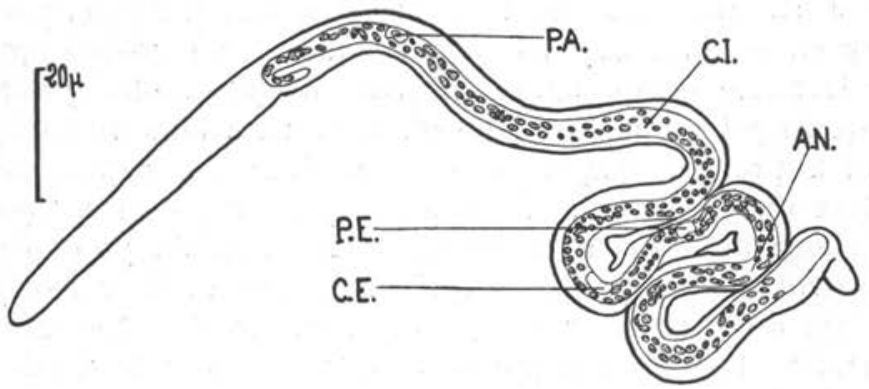

Fig. 2. - Macdonaldius andersoni: Microfilaire
A.N. : anneau nerveux
C.E. : cellule excrétrice
C.I. : corps interne
P.A. : pore anal
P.E. : pore excréteur

Les cellules rectales n'ont pas été repérées avec certitude. Le corps présente des courbures irrégulières, sans ondulations secondaires. Il est entouré d'une gaine qui, habituellement, dépasse largement chaque extrémité. La tête est arrondie, l'espace céphalique remar- 
quablement grand. Le corps interne n'est pas coloré, mais sa localisation est assez facile, car les noyaux somatiques sont, à son niveau, plus clairsemés. Au niveau de l'extrémité caudale, existent deux ou trois noyaux bien séparés les uns des autres, le dernier étant situé à environ $5 \mu$ de la pointe caudale. Celle-ci est assez fine, mais pas très pointue.

\section{Discussion}

En 1949, Ryerson a signalé brièvement la présence de microfilaires chez deux Heloderma suspectum sur neuf, et la récolte de vers adultes dans le cœur et l'aorte de l'un d'eux. Il est vraisemblable qu'il s'agit de la même espèce que la nôtre ; à notre connaissance, aucune description ou tentative de détermination n'a été publiée.

L'espèce appartient au genre Macdonaldius Khanna 1933 et se rapproche beaucoup de celle que nous avons décrite récemment sous le nom de $M$. oschei. Elle s'en distingue cependant par un bon nombre de petits caractères. L'espèce étudiée ici a une capsule buccale plus épaisse, un œsophage plus court, une vulve plus postérieure. Le sphincter prévulvaire est de forme différente. La queue du mâle est plus courte. L'ornementation cuticulaire ventrale en avant du cloaque est formée de crêtes transversales simples et non de séries de petites stries longitudinales. Le spicule gauche est plus court et le droit moins nettement ailé. Nous pensons donc que l'espèce est nouvelle et nous proposons le nom de Macdonaldius andersoni n. sp., pour la dédier à notre excellent ami Roy Anderson, à qui l'on doit tant d'acquisitions importantes sur les Filaires.

Le nombre des espèces incluses dans le genre se trouve porté à cinq :

Macdonaldius seetai Khanna 1933, d'un Coluber nord-américain, a été trouvée dans la veine porte. L'espèce est de grande taille (femelles jusqu'à $85 \mathrm{~mm}$.), les spicules très inégaux $(620 \mu$ et $150 \mu)$, les microfilaires longues $(320-390 \mu)$. C'est la seule espèce chez laquelle on ait signalé un petit gubernaculum.

M. carinii Vaz et Pereira 1935, d'un Elapomorphus (Colubridé) brésilien, a été trouvé dans le cœur. L'œsophage est relativement long $(800-980 \mu)$, les spicules courts $(220-260 \mu$ et $140 \mu)$. C'est la seule espèce qui ait des microfilaires très courtes (41-66 $\mu$ ).

M. grassii (Caballero 1954), d'un Sceloporus (Iguanidé) mexicain, a été signalée dans le péritoine, mais il serait intéressant de voir s'il n'y en a pas dans les vaisseaux sanguins. L'œsophage, relative- 
ment long, est faiblement divisé en deux parties. Les spicules sont courts $(266-296 \mu$ et $103 \mu)$; les microfilaires longues $(328 \mu)$. C'est la seule espèce qui ait une région péribuccale différenciée en plaque ovalaire.

M. oschei Chabaud et Frank 1961 a été récoltée chez des Pythons du Parc Zoologique de Stuttgart, mais il est vraisemblable que l'hôte d'origine est un Reptile du nouveau monde. Les adultes sont localisés dans les artères. Les spicules sont inégaux (455 et $120 \mu)$; les microfilaires de taille moyenne $(207 \mu)$. C'est la seule espèce dont la vulve soit pré-œsophagienne.

$M$. andersoni n. sp., d'un Héloderme nord-américain, vit également dans les artères. L'œsophage est court $(640 \mu)$, les microfilaires de taille moyenne $(218 \mu)$. Les spicules mesurent $310 \mu$ et $110 \mu$. La capsule buccale est un peu plus épaisse que celle des autres espèces.

\section{RÉSU MÉ}

Description de Macdonaldius andersoni n. sp., Filaire Onchocercidæ, qui vit dans les artères de l'Heloderma suspectum Cope, et y forme des pelotons qui semblent pouvoir bloquer la circulation sanguine. L'espèce est proche de M. oschei Chabaud et Frank 1961, mais s'en distingue par de nombreux petits caractères, et en particulier par la capsule buccale plus épaisse et plus chitinoïde.

\section{ZUSAMMENFASSUNG}

Beschreibung der Art Macdonaldius andersoni n. sp., einer Filarie, die zu den Onchocercidæ zu stellen ist. Die Filarien leben in den Arterien von Heloderma suspectum Cope 1869 und bilden Knäuel, die anscheinend die Blutzirkulation blockieren können. Die Art steht $M$. oschei Chabaud et Frank 1961 nahe, unterscheidet sich von ihr aber durch zahlreiche kleine Merkmale, besonders im Bau der Buccalkapsel.

\section{Bibliographie}

Caballero (E. C.), 1954. - Nematodes de los Reptiles de Mexico. XI. Nueve genero y nueva especie de Filaria de Iguanidos. Rev. Parasitol., XV, 305-313.

Chabaud (A.-G.) et Frank (W.), 1961. - Nouvelle Filaire parasite des Artères de Pythons: Macdonaldius oschei n. sp. (Nematodes Onchocercidæ). Zeitsch. f. Parasit., XX, 434-439. 
Golvan (Y.), 1957. - Les principales techniques de coloration des Microfilaires sanguicoles. Bull. Soc. Pathol. Exot., L, 143-157.

Khanna (R. K.), 1933. - A new filarial worm from a North American Snake. J. Helmint., XI, 105-108.

Ryerson (D. L.), 1949. - A preliminary survey of reptilian blood. J. Ent. zool. Claremont, Cal. XLI, 49-55.

VAz (Z.) et Pereira (C.), 1935. - Some new Brazilian Nematodes. Trans. Micros. Soc., LIV, 36-40.

(Institut de Parasitologie, Faculté de Médecine de Paris et Zoologisches Institut Stuttgart - Hohenheim)

\section{NOTE ADDITIONNELLE}

\section{Description de la microfilaire de Macdonaldius oschei}

\section{Par Alain-G. CHABAUD et Werner FRANK}

La description de Macdonaldius oschei, que nous avons publiée en 1961 dans $Z$. f. Parasitenkunde, XX, p. 434-439, indique seulement que les « microfilaires paraissent dépourvues de gaine et sont longues d'environ $200 \mu »$.

Depuis cette époque, de nouvelles infestations ont été observées au Parc Zoologique de Stuttgart chez certains Python reticulatus (Schneider 1801) et Constrictor constrictor (L.) ; ce dernier constituant ainsi un nouvel hôte chez lequel des adultes ont été récoltés. Il a été possible également d'obtenir chez ces différents Serpents des frottis de sang plus ou moins riches en microfilaires. N'ayant pas de frottis de l'hôte type, $P$. bivittatus, nous décrivons ici les microfilaires du sang de $P$. reticulatus $\left(\mathrm{n}^{\circ} 2\right.$ du 12-V-1961), colorées par le Giemsa, selon la technique habituelle.

\section{Description}

La microfilaire est incluse dans une enveloppe ovalaire qui correspond évidemment à une gaine, mais celle-ci, bien qu'elle soit mince et souple, n'épouse pas les formes de la microfilaire comme il est habituel. Elle forme une sorte d'œuf ovalaire, plus ou moins aplati, à l'intérieur duquel la microfilaire ne peut s'étendre complètement. Elle y présente des courbures simples ou, assez souvent, y est repliée sur elle-même, pour prendre la forme d'un V ou d'un C.

Les dimensions moyennes observées sur cinq spécimens sont : Longueur : $207 \mu$; largeur au niveau de l'anneau nerveux : $6 \mu$. Anneau nerveux, pore excréteur et cellule excrétrice situés respecti- 


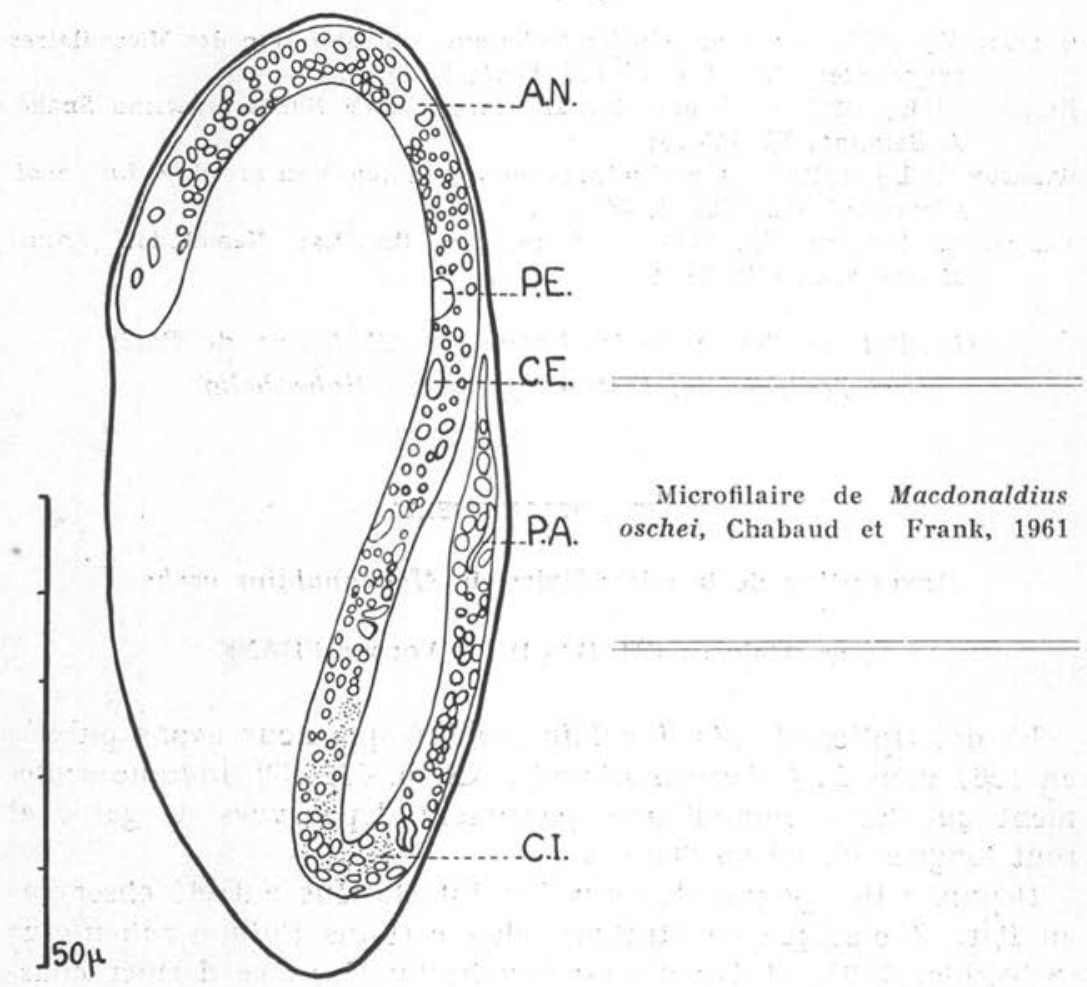

vement à $50 \mu, 72 \mu$ et $83 \mu$ de l'apex. Corps interne long de $34 \mu$, avec sa partie centrale située à $142 \mu$ de l'extrémité antérieure. Pore anal à $14 \mu$ du noyau le plus postérieur.

L'espace céphalique, généralement un peu dilaté, est pauvre en noyaux. Le premier de ceux-ci est isolé à une distance variable de l'apex (de 4 à $12 \mu$ ); il est suivi de un ou deux noyaux généralement séparés de la colonne des noyaux sous-cuticulaires et somatiques. Ceux-ci sont assez petits et généralement bien séparés les uns des autres. La cellule excrétrice et le pore excréteur sont grands et bien visibles; ils sont proches l'un de l'autre. Le corps interne se colore facilement par le Giemsa ; il est constitué par un nuage de petites granulations rouges qui n'interrompt pas complètement la colonne nucléaire. Les cellules rectales sont peu apparentes. Il semble y en avoir deux juste en arrière du corps interne et deux autres plus postérieures et plus espacées. Le pore anal est souvent marqué par une petite tache colorée, de forme allongée. La pointe caudale est constituée par une colonne de 4 ou 5 noyaux régulièrement alignés et se prolonge parfois par une petite pointe hyaline, longue d'environ $5 \mu$. 